\title{
Network Adaptive Interference Aware Routing Metric For Hybrid Wireless Mesh Networks
}

\author{
Ubaid Ullah and Adnan K. Kiani \\ School of Electrical Engineering and Computer Science, \\ National University of Sciences and Technology, \\ Islamabad, Pakistan. \\ Email: \{ubaid.ullah, adnan.khalid\}@ seecs.edu.pk
}

\author{
Raja Farrukh Ali and Rizwan Ahmad \\ School of Electrical Engineering and Computer Science, \\ National University of Sciences and Technology, \\ Islamabad, Pakistan. \\ Email: \{farrukh.ali, rizwan.ahmad\}@ seecs.edu.pk
}

\begin{abstract}
Wireless Mesh Networks provide a reliable, robust and resilient platform for broadband access. Main benefits of using Wireless Mesh Networks are their low cost, robustness, self healing, and self configuring properties. In Wireless Mesh Networks, routing metric determines the path from source to destination. Wireless link conditions can be affected by a number of factors including interference, congestion, mobility, and network topology. Routing metric needs to consider all these factors while making routing decisions. In addition, wireless link conditions do not remain static with time requiring the routing metric to be adaptive. Interference in Wireless Mesh Networks are of two types: inter-channel and intra-channel interference. Existing routing metrics for Wireless Mesh Networks either consider only one of the two interference types or do not capture changing network conditions. In this paper, we propose a new routing metric for Wireless Mesh Networks which takes into account both inter and intra-channel interference and is adaptive to changing network conditions. Our proposed metric is compared with the state of the art and shows throughput improvement of up to 20 percent and latency reduction of 25 percent.
\end{abstract}

Keywords-Wireless mesh networks, multi-radio, routing metric, load aware routing, performance

\section{INTRODUCTION}

With the emergence of a number of bandwidth hungry always on broadband Internet applications, wireless access is required to be robust, reliable and have good connectivity. One of the candidates to provide wireless access is Wireless Mesh Network (WMN). WMNs are of three types [1]: Infrastructure backbone, client backbone and hybrid WMNs. Out of these, hybrid WMNs are the most prevalent as they combine the connectivity pattern of both Infrastructure and client WMNs. In addition, they are dynamic, self-organizing and self-healing networks which enable nodes to establish and maintain connectivity. Hybrid WMNs comprise of two types of nodes: mesh clients and mesh routers. Mesh clients are generally small battery mobile nodes equipped with a single radio and extend reach of network. The connectivity to mesh clients is provided by mesh routers. Mesh routers form the basic backbone of hybrid WMNs. They are static in nature and are equipped with multiple radios. In addition to providing mesh routing functionality, mesh routers are used to provide bridge/gateway function. Hybrid WMNs provide reliable and good connectivity for a wide range of applications which was not possible with other wireless networks such as cellular, sensor and pure ad hoc. Mesh routers can significantly enhance network connectivity through utilizing multiple orthogonal channels.
However, despite their widespread popularity, WMNs face challenges in their large scale deployment. These challenges include Interference, mobility, link congestion, and network load. A number of routing protocols have been proposed to overcome these challenges.

Routing protocols are the heart of WMNs as they control the formation, configuration, and maintenance of routes between source and destination. Depending upon nature of the network, routing protocols are designed to utilize the resources in a better way and to maintain connectivity amongst nodes. In static networks the emphasis is on improving the performance of individual transfers [1], while a mobile network aims to employ protocol which can maintain connectivity in face of frequent node movement [2]. Routing protocols employ routing metrics to determine best path between source and destination. A routing metric that accurately captures quality of network links is central to computation of good quality paths. There can be many factors affecting the wireless link. These include mobility, link congestion, network topology, and nodes residual energy. As a result, there are a number of parameters based on which a routing metric algorithm can make decisions. These parameters include but are not limited to hop count, throughput, channel congestion, interference, and link load [3]. A routing metric can base its decision on a single parameter or can combine them by assigning weights to each. Traditional ad hoc networks considered hop count as the soul parameter for making routing decisions. However, it was shown in [4] that considering only hop count resulted in discovering longer, slower paths. Subsequently, some metrics have been proposed which consider factors of packet loss, channel congestion and inter-channel interference amongst others. These metrics tend to find shorter paths with lower end to end delay while reducing intra-channel interference resulting in effective utilization of network resources.

Interference is one of the primary challenges in the way of large scale WMNs deployment. In WMNs settings, two types of interference can occur: Inter-channel interference and intra-channel interference. Inter-channel interference refers to the interference between neighboring routers competing for the same busy channel. Intra-channel interference refers to the interference between routers on the same path/flow operating on the same channel. Inter-channel interference is harder to control owing to the involvement of multiple flows and routes. There have been a few metrics proposed in literature which tackle Interference in WMNs. Although these 
metrics provide some measure of relief, they all have some inherent shortcomings such as non-isotonicity, measurement complexity, and tackling only one of the two interference types, inter-channel or intra-channel interference. In addition, a wireless medium requires routing metric to be able to adapt itself to dynamic nature of the network and be reflective of the current network conditions. Set of currently used metrics fail o fulfill this requirement. They make decisions based on a number of factors by assigning weights to them. This is done through introducing a tunable factor which cannot be changed dynamically [4] [5]. Thus current network conditions are not truly captured while making routing decisions. In one of our earlier works, we presented D-WCETT routing metric which used Interface Queue Length to determine channel load and made necessary adjustments to tunable factor [6]. We saw marked improvements when D-WCETT was compared to its predecessors. In making the routing decisions $D$-WCETT ignored inter-channel interference levels. In this paper, we present an interference aware dynamic routing metric which takes into account current link conditions as well as interchannel interference. We call the metric Network Adaptive Interference Aware (NAIA). The performance of NAIA is evaluated in terms of its adaptability to changing network conditions and interference. We implemented NAIA in multiradio hybrid WMNs and compared the performance with WCETT and D-WCETT. The results showed improvement in terms of throughput gain and latency while there was a slight increase in routing packet overhead. The rest of the paper is organized as follows: Section II describes the related work on interference capturing routing metrics. In section III our proposed routing metric NAIA is presented. Section IV presents the simulation setup and results followed by conclusion in section V.

\section{RELATED WORK}

Hop count is the most commonly used routing metric for traditional infrastructureless networks. However, the problems of using hop count as routing metric have been well documented [4]. The main concern is that it ignores data transmission rates and link quality. This results in inefficient path selection and degraded network performance. Expected Transmission Count (ETX) [7] routing metric is defined as the number of transmissions required to successfully deliver a packet over wireless link. ETX takes into account link conditions as it favors paths with higher throughput. Probe packets are used to find forward and reverse delivery ratios. Although, ETX provides better performance compared to hop count, it is mainly a single-channel multi-hop solution. It does not capture the true link conditions in multi-channel environment. It also ignores Interference experienced on wireless links. In addition, probe packets are transmitted at much lower rates than actual data packets resulting in false delivery ratios. Expected Transmission Time (ETT) [7] is another routing metric presented for infrastructureless networks. It is given by the formula:

$$
E T T=E T X \cdot \frac{S}{B}
$$

Where $S$ is the packet size and $B$ is bandwidth of the channel. ETT is essentially the expected time to successfully transmit a packet at MAC layer. ETT path metric is calculated by adding all the ETT values on individual links along the path. ETT is more efficient than ETX as it takes packet size and link capacity into account. However, it retains some of the disadvantages of ETT. There is again no support for multi-radio networks and hence no provision to provide channel diversity. The resultant paths may have high levels of interference. There is also no support to dynamically capture the current link conditions or network load.

Weighted Cumulative Expected Transmission Time (WCETT) [4] is the multi-radio extension of ETT. The main advantage of using multiple radios is to increase capacity by using multiple orthogonal channels for transmissions. This inherently provides channel diversity. The WCETT metric value for a path $\mathrm{p}$ is given by:

$$
W C E T T_{p}=(1-\beta) \cdot \sum_{l \in p} E T T_{l}+\beta \cdot \max _{1 \leq j \leq k} X_{j}
$$

Where the term $E T T_{l}$ is the sum ofETTs on all the links along the path $p$ and $\max X_{j}$ is the summation of all ETTs on the most consumed channel while k represents the total number of channels available in the network. Beta $(\beta)$ is a tunable parameter which allocates weights to path length and channel diversity and is fixed throughout the operation of network. The first term of WCETT selects path with minimum ETT while the second term indicates channel diversity helping intra-channel interference reduction. Although WCETT offers improvement over ETT and partially addresses the problem of intra-channel interference, there are still some shortcomings especially in the context of hybrid WMNs. A major limiting factor of WCETT is that it does not capture inter-channel interference while forwarding data. Inter-channel interference occurs when two nodes lying in the carrier sensing range of one another and transmitting at the same time using the same channel. WCETT is also non-isotonic. Isotonic property of a metric ensures that order of the weights of the two paths are preserved when they are appended by a common third path. Isotonic is a necessary condition for finding minimum path weight paths and to ensure loop free routing [8], [9].

Metric of Interference and Channel Switching (MIC) [10] is designed specifically to address the problem of interference in WMNs. It also provides load balancing. MIC for a path $p$ is given by the formula:

$$
M I C(p)=\frac{1}{\min (E T T) \cdot N} \sum_{l} I R U_{l}+\sum_{i} C S C_{i}
$$

Where $N$ represents the total number of nodes in the network. While the two components of $M I C$ are separately given by the formulae:

$$
I R U_{l}=E T T_{l} \times N_{l}
$$

and

$$
C S C_{i}= \begin{cases}w 1 & \text { ifCH(prev }(i)) \neq C H(i) \\ w 2 & \text { ifCH(prev }(i))=C H(i)\end{cases}
$$

Where $N_{l}$ is the number of interfering neighbors with the link $l . C H(i)$ is the channel allocated to the node $i$ and prev(i) is the channel used in the previous hop. The first term IRU caters for the inter-channel interference while the second term 
deals with intra-channel interference. Main disadvantage of using $M I C$ is that that there is a lot of overhead associated with maintaining and updating values of the ETTs for each link. This can significantly affect network performance. MIC also assumes that all links in the collision domain of a particular link contribute to the same level of interference. This is not always true as there might be no transmissions taking place on some of the links. There is also no provision for making the metric adaptable to changing network conditions.

iAware routing metric [11] is specially designed for multiradio WMNs which considers intra-channel and inter-channel interference while forwarding data within network. It captures physical interference at a node. iAware metric is defined as:

$$
\text { iAware }_{p}=(1-\alpha) \cdot \sum_{j=1}^{n} \text { iAware }_{i}+\alpha \cdot \max _{1 \leq j \leq k} X_{j}
$$

$X_{j}$ is same as in WCETT. The iAWARE value of a link $j$ is defined as follows:

$$
i_{\text {Aware }}=\frac{E T T_{j}}{I R_{j}}
$$

Where $I R_{j}$ is interference ratio which is defined as:

$$
I R_{j}=\min \left[I R_{j}(u) I R_{j}(v)\right]
$$

And $I R_{j}(u)$ is defined as:

$$
I R_{j}(u)=\frac{S I N R_{j}(u)}{S N R_{j}(u)}
$$

iAware does not capture the changing link conditions as $\alpha$ remains static and is only calculated at the start. Although both inter-channel and intra-channel interference are taken into account, iAware is non-isotonic. Additionally, for cases where a link has high $I R$ value resulting in low metric value, iAware will choose paths with low ETT and high interference values.

We have presented $D$-WCETT in [6]. D-WCETT addresses the problem of static tunable factor in WCETT. In D-WCETT the value of $\beta$ is dynamic and is depended on interface queue length (IFQ).

$$
W C E T T_{p}=(1-\beta) \cdot \sum_{l \in p} E T T_{l}+\beta \cdot \max _{1 \leq j \leq k} X_{j}
$$

$I F Q$ length is a parameter indicating the total number of packets in the queue of a channel belonging to a node. IFQ is basically a drop tail buffer. The build up of frames indicates contention among nodes resulting in congestion. IFQ value is locally available on the node and requires no calculations. $I F Q$ is normalized through equation:

$$
Q D I=\frac{I F Q}{B W}
$$

Where $Q D I$ is the Queue Discharge Interval which is the time required to discharge the data present in the queue of a node. $B W$ represents the bandwidth of the link between the two nodes. More the value of IFQ more will be the value of $Q D I S$ and vice versa. $\beta$ is given by the equation:

$$
\beta=1-Q D I
$$

Value of $\beta$ remains between 0 and 1. When large queues are experienced on a particular channel, D-WCETT metric
TABLE I: Pseudo code for interference calculation

\begin{tabular}{|l|l|}
\hline S.No & \multicolumn{1}{|c|}{ NAIA Algorithm } \\
\hline 1 & $\begin{array}{l}\text { Initialize } d, C, S N R, \text { SINR, } N, \text { Int } \\
\text { Measure distance } d \text { of } N \text { from all the near-by nodes } \\
\text { while receiving packets } \\
\text { If (Nodes Distance } d \leq \text { Carrier Sensing Range } \\
C)\end{array}$ \\
& $\begin{array}{l}\text { Declare the node as interfering node } \\
\text { Else }\end{array}$ \\
4 & $\begin{array}{l}\text { Declare the node as non-interfering node } \\
\text { End if } \\
\text { Measure SNR and SINR from all the interfering } \\
\text { nodes at node } N \text { and evaluate interference Int on } \\
\text { associated links. }\end{array}$ \\
& \\
\hline
\end{tabular}

switches transmission to a different channel thus providing channel diversity. $D$-WCETT provides much improved performance compared to WCETT. However, D-WCETT does not take into account inter-channel interference.

\section{NETWORK AdAPTIVE INTERFERENCE AWARE METRIC (NAIA metric):}

When it comes to multichannel environments there are two types of interference that can be experienced by a packet; Inter-channel and Intra-channel interference. Further improvements can be achieved when inter-channel interference is incorporated in routing decisions. Our work has two major contributions; (i) inter-channel interference calculations and (ii) evaluation of the value of proposed metric NAIA. Pseudo code for Interference Calculations is given in Table I. Next subsections present details of the contributions.

\section{A. Inter-Channel Interference Calculation:}

Inter-channel interference at a node is calculated by taking into account the distance between two nodes. The nodes in the carrier sensing range can cause inter-channel interference. The transmission range $\mathrm{D}$ is given by the formula [12].

$$
D=\frac{P_{t} \times \lambda^{2}}{P_{r} \times 4 \pi^{2}}
$$

Where $P_{t}$ and $P_{r}$ are the transmission and reception power of the node respectively. $\lambda$ is the ratio of speed of light $(c)$ to node operating frequency $(f)$ that is $\lambda=c / f$. Carrier sensing range $C$ is approximately 1.5 times the transmission range [13]. The distance between two nodes is calculated by Euclidean formula given below:

$$
d=\sqrt{\left(Z_{2}-Z_{1}\right)^{2}+\left(Y_{2}-Y_{1}\right)^{2}+\left(X_{2}-X_{1}\right)^{2}}
$$

Where $d$ is the distance between two nodes having coordinates $\left(Z_{2}, Z_{1}\right),\left(Y_{2}, Y_{1}\right)$ and $\left(X_{2}, X_{1}\right)$ respectively. Inter channelinterference will occur if two nodes lying in carrier sensing range start transmitting at the same time using the same channel. While nodes inside transmission range defer their transmissions after sensing another node's data. Depending 
upon the distance, neighboring nodes are declared either interfering or non-interfering. Interference from all interfering nodes is summed at a receiver (node $N$ ) and interference ratio $(I R)$ [14] is calculated by using equation 9 which is the interference on a link and we have taken an aggregated value of all the associated links. The inter-channel interference at a link can be calculated by taking the inverse of the IR. The formula is:

$$
\text { Int }=\frac{1}{I R}
$$

The sender node compares interference level on each link while making routing decisions. Load at every link is also given weightage while forwarding packets. The selected path is a tradeoff between load balancing and interference. If the interference is less than a set threshold, the link or channel is not switched. This is done to avoid continuous switching. Continuous switching leads to frequent changes in metric. Pseudo code for our algorithm is given in Table I.

\section{B. Network Adaptive Interference Aware Metric (NAIA) cal- culation:}

The new metric NAIA is defined by formula:

$$
N A I A_{p}=(1-\beta) \cdot i n t+\beta \cdot \max _{1 \leq j \leq k} X_{j}
$$

Where int is inter-channel interference and $X_{j}$ is the summation of the ETTs at most consumed channel. The first part of the metric caters for inter channel interference while the second part ensures use of different channels at consecutive hops thus avoiding intra-channel interference. Value of $\beta$ is obtained from equation 12. Lower value of $\beta$ assign more weightage to inter-channel interference while greater value of $\beta$ gives more importance to channel diversity. When there is more load on the link lower $\beta$ value is obtained. In case of low load, path with low inter-channel interference is chosen. Furthermore, NAIA is isotonic. We have evaluated NAIA through running a series of simulations. The results are presented in next section.

\section{PERFORMANCE EVAlUATION OF NAIA:}

\section{A. Simulation Environment:}

In order to gauge the performance of NAIA a series of simulations were run using NS-2 [15]. AODV was used as a routing protocol [16]. Two sets of simulation were run, with one set of results obtained by varying packet transmission rate and the other set by varying number of concurrent flows. Concurrent UDP flows were generated between randomly selected source and destination pairs. After conducting a number of experiments we fixed the $I F Q$ window size at 0.1 seconds which gave us optimal results. The default simulation parameters are given in Table II.

\section{B. Performance Metrics:}

The following three performance metrics were considered:

- Throughput Gain: It is increase in throughput compared to WCETT.
TABLE II: Simulation Parameters.

\begin{tabular}{|l|l|}
\hline & WCETT,D-WCETT and NAIA \\
\hline Simulation Area & $1000 \times 1000 \mathrm{~m}$ \\
Simulation Time & 900 seconds \\
Propagation Model & Two-Ray Ground Reflection \\
MAC protocol & 802.11 DCF \\
physical layer & $802.11 \mathrm{~b}$ \\
Routing Protocol & AODV \\
Mobility Model for Mesh Clients & Random Waypoint \\
No. of Mesh Routers & 25 \\
No. of Mesh Clients & 50 \\
Traffic Type & CBR (UDP) \\
Packet Size & 512 bytes \\
Packet Transmission Rate & 32 packets/sec \\
No. of Sources (Flows) & 30 flows \\
Max No. of Packets in $I F Q$ & 50 packets \\
$I F Q$ Window Size & $100 \mathrm{~ms}$ \\
& \\
\hline
\end{tabular}

- $\quad$ Routing Packet Overhead: The ratio of control packets generated and number of successfully received data packets.

- Average Latency : The mean time (in seconds) taken by data packets to reach their respective destinations.

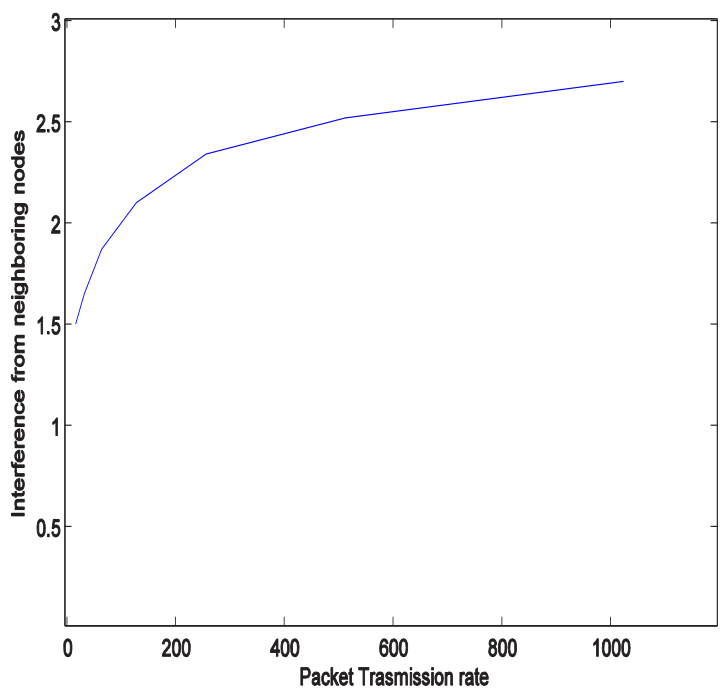

Fig. 1: Packet Transmission Rate Vs. Interference Ratio

1) Varying Packet Transmission Rate: In this set of simulations packet transmission rate was varied from 16 to 1024 packets/sec while keeping number of concurrent flows constant at 5. Interference versus packet transmission rate is plotted in Fig. 1. Interference increases with packet transmission rate. The reason for this is high traffic congestion at higher transmission rates. From the Fig it can observed that at transmission rate of 600 packets/sec the quantitative value of interference is 2.4. This means $S I N R$ obtained is 2.4 times greater than $S N R$ received at a node.

Throughput gain over WCETT versus packet transmission rate is shown in Fig. 2a. Throughput gain increases as the packet transmission rate is increased. At 512 packets/sec NAIA and D-WCETT show improvement over WCETT of 20 and 


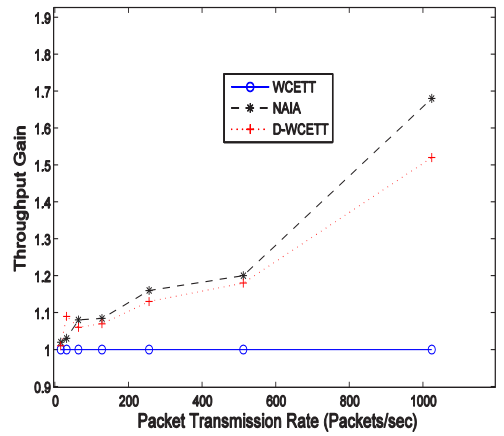

(a) Packet Transmission rate vs. Throughput gain

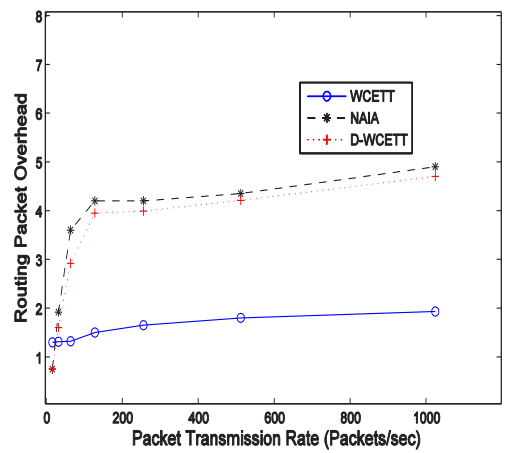

(b) Packet transmission rate vs. Routing overhead

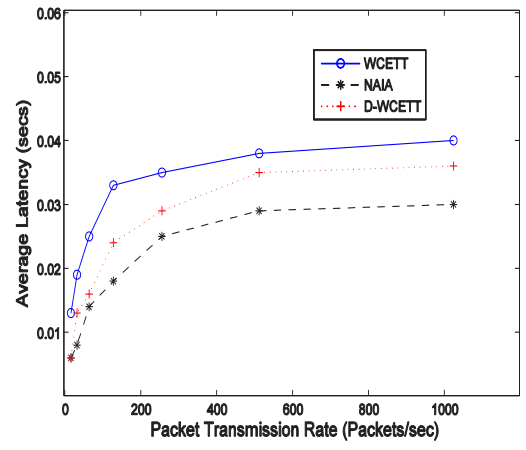

(c) Packet transmission rate vs. Latency

Fig. 2: Simulation results of varying packet transmission rate

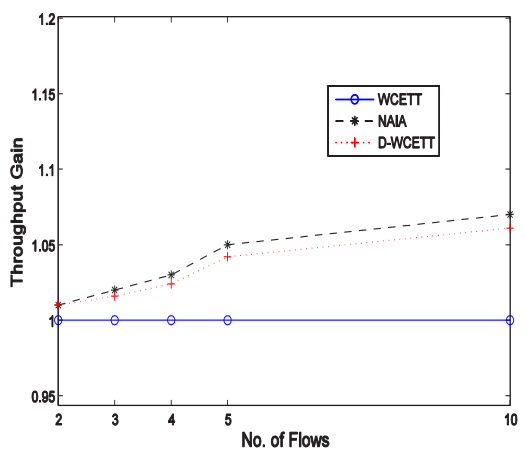

(a) No. of flows vs. Throughput gain

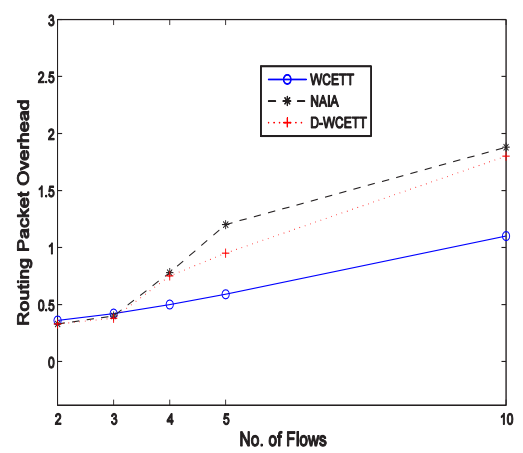

(b) No. of flows vs. Routing Packet Overhead

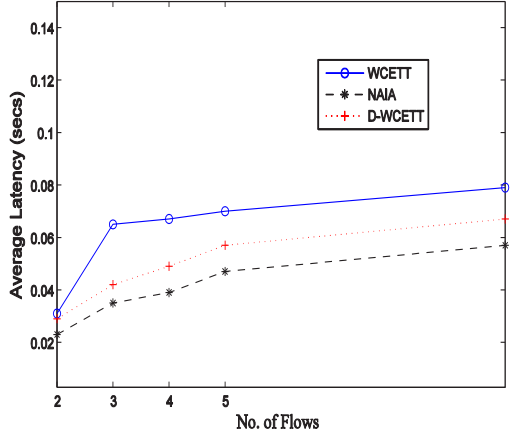

(c) No. of Flows vs. Average Latency

Fig. 3: Simulation results of varying number of flows

15 percent respectively. The improvements are more profound at higher packet transmission rate. An interesting result is obtained at 16 packets/sec where $D$-WCETT outperforms both NAIA and WCETT. This can be attributed to the fact that $D$ WCETT balances between channel diversity with path length. At low packet transmission rate inter-channel interference has negligible affect. Hence $D$-WCETT performance is better. As packet transmission rate increases, NAIA starts to outperform both WCETT and D-WCETT. This is due to NAIA's interference aware property avoiding links with increased interference.

Packet transmission rate vs. routing packet overhead is shown in Fig. 2b. At low packet transmission rates routing overhead for all metrics remains low. When transmission rate increases, routing overhead shows a significant increase. The trend can be observed in going from 16 packets/sec to 64 packets/sec. This is due to adaptive nature of NAIA and D-WCETT. Over the course of transmission it is possible that more optimal paths are discovered resulting in additional RREQ packets and subsequently control packets storm. From 64 packets/sec onwards the control packet increase is negligible. This is because of large amount of packets dropped due to buffer overflows. This results in reduce route discovery resulting in negligible increase in routing overhead.

Average latency versus packet transmission rate is given in Fig. 2c. Packets experience low latency when NAIA is employed. NAIA selects paths with least interference which results in quicker delivery of packets. The latency experienced in case of NAIA is less than D-WCETT and WCETT because of interference aware and adaptive nature of NAIA. NAIA shows 25 and 16 percent improvement over WCETT and D-WCETT respectively.

2) Varying Number of Flows: Number of concurrent flows were varied from 2 to 10 while keeping router to client ratio 1:3. Results are shown in the Fig. 3. Throughput gain versus number of flows is shown in Fig. 3a. Throughput gain increases with number of concurrent flows. NAIA gives maximum throughput gain of 7 and 2 percent over WCETT and $D$-WCETT respectively. Reason for this is NAIA's interference aware capability which ensures that as inter-channel interference increases, paths offering least interference are chosen. Throughput gain shows greater increase upto 5 concurrent flows. From 5 onwards the gain is more linear. This can attributed to the fact that upto 5 flows the link congestion levels increase rapidly. After 5 flows the congestion increase is more steady resulting in more linear curve.

Routing packet overhead versus number of concurrent flows is shown in Fig. 3b. As the number of flows increase, routing overhead also increases. It's levels are low at start and show a steep increase upto 5 concurrent flows. The increase in routing overhead is more linear from 5 onwards. 
Upto 3 flows the routing overhead experienced by all three metrics are the same. From 3 flows onwards NAIA has greater routing overhead. This is because in NAIA more RREQ packets traverse the network as there are more updates due to changing nature of links. In addition NAIA also caters for inter-channel interference. The routing overhead for $D$-WCETT remains below NAIA. Network becomes more congested when number of concurrent flows increase. Catering congestion in the network demands more control packets generation.

Fig. $3 c$ gives the average latency versus number of flows. WCETT offers highest average latency due to its inability to cater for load balancing and interference. This is followed by $D$-WCETT as it only caters for intra-channel interference. NAIA takes into account both inter and intra-channel interference and selects channel with least interference levels. This results in low latency.

\section{CONCLUSION AND FUtURE WORK}

This paper presents an interference catering metric NAIA for hybrid wireless mesh networks. The proposed metric caters for intra-channel and inter-channel interference. Paths are selected on the basis of load and interference levels. The path is selected dynamically depending upon the value of interface queue length, which is a direct indication of the link load at a node. NAIA is able to incorporate inter-channel interference while making routing decisions. It balances load and interference dynamically. NAIA metric is compared with both $D$-WCETT and WCETT by running a series of simulations. The results show improvement in terms of throughput gain and average latency while there is a slight increase in routing overhead.

In future we plan to include mobility in our analysis. Mobility directly affects link stability. We also look to analyze our metric by using different router to client ratios.

\section{REFERENCES}

[1] I. F. Akyildiz, X. Wang, and W. Wang, "Wireless mesh networks: a survey," Computer Networks, vol. 47, no. 4, pp. 445-487, 2005.

[2] R. Baumann, S. Heimlicher, M. Strasser, and A. Weibel, "A survey on routing metrics," TIK Report, vol. 262, 2007.

[3] L. Zhao and A. Y. Al-Dubai, "Routing metrics for wireless mesh networks: a survey," in Recent Advances in Computer Science and Information Engineering. Springer, 2012, pp. 311-316.

[4] R. Draves, J. Padhye, and B. Zill, "Routing in multi-radio, multihop wireless mesh networks," in Proceedings of the 10th annual international conference on Mobile computing and networking. ACM, 2004, pp. 114-128.

[5] Y. Zhao, H. Wang, Y. Li, and H. Song, "A multi-channel routing protocol for dual radio wireless networks," in High Performance Computing and Communications \& 2013 IEEE International Conference on Embedded and Ubiquitous Computing (HPCC_EUC), 2013 IEEE 10th International Conference on. IEEE, 2013, pp. 2271-2276.

[6] R. F. Ali, A. K. Kiani, and A. A. Pirzada, "Load dependent dynamic path selection in multi-radio hybrid wireless mesh networks," in Wireless Communications and Networking Conference (WCNC), 2014 IEEE. IEEE, 2014, pp. 2020-2025.

[7] D. S. De Couto, D. Aguayo, J. Bicket, and R. Morris, "A highthroughput path metric for multi-hop wireless routing," Wireless Networks, vol. 11, no. 4, pp. 419-434, 2005.

[8] Y. Yang, J. Wang, and R. Kravets, "Designing routing metrics for mesh networks," in IEEE Workshop on Wireless Mesh Networks (WiMesh), 2005 .
[9] V. Kisara, "A new routing metric for wireless mesh networks," 2010.

[10] Y. Yang, J. Wang, and R. Kravets, "Designing routing metrics for mesh networks," in Proc. of IEEE Workshop on Wireless Mesh Networks (WiMesh), 2005.

[11] A. P. Subramanian, M. M. Buddhikot, and S. Miller, "Interference aware routing in multi-radio wireless mesh networks," in Wireless Mesh Networks, 2006. WiMesh 2006. 2nd IEEE Workshop on. IEEE, 2006, pp. 55-63.

[12] T. S. Rappaport et al., Wireless communications: principles and practice. prentice hall PTR New Jersey, 1996, vol. 2.

[13] K. Jamieson, B. Hull, A. Miu, and H. Balakrishnan, "Understanding the real-world performance of carrier sense," in Proceedings of the 2005 ACM SIGCOMM workshop on Experimental approaches to wireless network design and analysis. ACM, 2005, pp. 52-57.

[14] A. P. Subramanian, M. M. Buddhikot, and S. Miller, "Interference aware routing in multi-radio wireless mesh networks," in Proc. of 2nd IEEE Workshop on Wireless Mesh Networks (WiMesh), 2006, pp. 55-63.

[15] NS, "The Network Simulator," http://www.isi.edu/nsnam/ns/, 1989.

[16] C. E. Perkins and E. M. Royer, "Ad-hoc on-demand distance vector routing," in Proc. of 2nd IEEE Workshop on Mobile Computing Systems and Applications, 1999, pp. 90-100. 